$\therefore \quad$ LA-UR- $9 \eta-1855$

Title:

Author(s):

Submitted to:

\section{3}

CONF-971154_-

TRANSIONOSPHERIC SIGNAL DETECTION WITH CHIRPED WAVELETS

RECENED

\section{AUG 131997 \\ OSTI}

Mark E. Dunham, NIS-1

Asilomar Conference

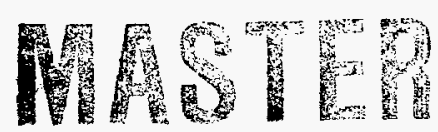

Monterey, CA

Nov 2-5, 1997

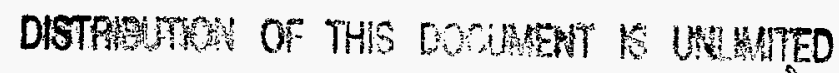
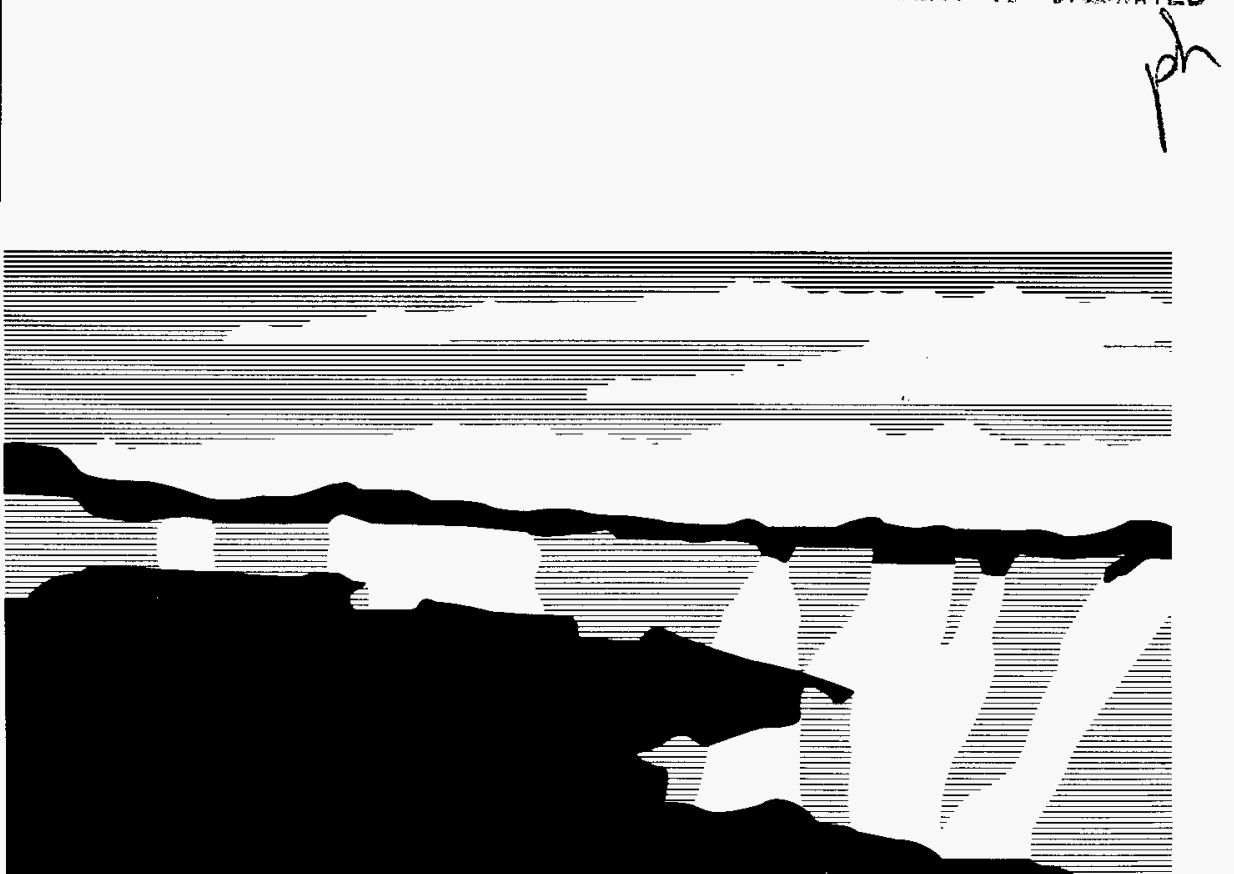


\section{DISCLAIMER}

This report was prepared as an account of work sponsored by an agency of the United States Government. Neither the United States Government nor any agency thereof, nor any of their employees, make any warranty, express or implied, or assumes any legal liability or responsibility for the accuracy, completeness, or usefulness of any information, apparatus, product, or process disclosed, or represents that its use would not infringe privately owned rights. Reference herein to any specific commercial product, process, or service by trade name, trademark, manufacturer, or otherwise does not necessarily constitute or imply its endorsement, recommendation, or favoring by the United States Government or any agency thereof. The views and opinions of authors expressed herein do not necessarily state or reflect those of the United States Government or any agency thereof. 


\title{
Transionospheric Signal Detection with Chirped Wavelets
}

\author{
Adele B. Doser (POC) \\ Mark E. Dunham \\ Non-Proliferation and International Security Division, M/S D466 \\ Los Alamos National Laboratory \\ Los Alamos, New Mexico 87545
}

5056654111

5056657395 (FAX)

adoser@lanl.gov

5056670045

Keywords: 3. Time/frequency representations, wavelets, filter banks

\begin{abstract}
Chirped wavelets are utilized to detect dispersed signals in the joint time/scale domain. Specifically, pulses that become dispersed by transmission through the ionosphere and are received by satellites as nonlinear chirps are investigated. Since the dispersion greatly lowers the signal-to-noise ratios, it is difficult to isolate the signals in the time domain. Satellite data are examined with discrete wavelet expansions. Detection is accomplished via a template matching threshold scheme. Quantitative experimental results demonstrate that the chirped wavelet detection scheme is successful in detecting the transionospheric pulses at very low signal-to-noise ratios.
\end{abstract}

\section{Introduction}

Time/frequency methods can be useful tools for detecting signals that cannot be easily isolated in either time or frequency. Our interest lies primarily with the satellite-based detection of impulse signals, which are dispersed as they travel through the ionosphere, and appear as nonlinear chirps at the receiver [1]. The received pulses typically have very low signalto-noise ratios, which make them difficult to detect in the time or frequency domain. While the nonlinear chirps are apparent in the joint time/frequency domain, the computational complexity of traditional time/frequency methods renders them impractical for real-time applications, where typical sampling rates are $150 \mathrm{MHz}$ [2]. However, wavelet procedures are superior to traditional time/frequency methods in terms of computational efficiency. Because of the chirp present in the received signals, we examine the use of chirped wavelets $[3,4]$ in their detection.

Section 2 employs chirped wavelets in the discrete wavelet transform, applied to actual time series recorded by the US Department of Energy's ALEXIS/BLACKBEARD satellite. In section 3, the wavelet transform is enlisted in a template matching scheme to detect the recorded transionospheric signals. Experimental probability of detection and false alarm are used as performance measures. Finally, summary and conclusions are presented.

\section{Discrete Wavelet Expansion with Chirped Wavelets}

Figure (1) shows a weak signal recorded by a DOE radio science experiment aboard the ALEXIS/BLACKBEARD satellite, with a sampling rate of $150 \mathrm{MHz}$. The signal was produced using the Los Alamos portable pulser (LAPP), and was dispersed by the ionosphere so that it was received as a descending frequency nonlinear chirp. Due to noise and carrier interference, it is impossible to distinguish the LAPP chirp signal in the time series (the peak power of the signal is approximately $1 / 20$ that of the interference). The Fast Fourier Transform (Figure (2)) is not better. The large contribution at the top of the spectrum is not the LAPP, but interfering signals. However, in a time/frequency plot, such as the spectrogram of Figure (3), the weak LAPP signal is apparent as a nonlinear function. The displayed spectrogram has several thousand coefficients, and the LAPP signal is faint.

Discrete wavelet transforms were performed on the LAPP data. The analysis wavelets were produced using the general formula

$$
w(n)=\cos \left(a n^{p}\right) .
$$

The acceleration rate, $p$, and the parameter $a$ were varied in order to produce an ensemble of 45 wavelets, with $f_{h} / f_{l}$ (the ratio of the highest frequency present in $w$ to the lowest) ranging between 1 and several hundred. The time series was windowed with a Gaussian/Malvar combination window.

Discrete wavelet expansions of the LAPP signal from Figure (1) were performed using a dyadic decomposition. An example of the resulting scalograms is displayed in Figure (4). Seven scale levels are shown in the figure. The LAPP signal can be witnessed as a descending diagonal pattern in the figure. The noise in the highest scale is caused by the large ensemble in the FM band that can be seen at the high end of the spectrum in Figure (2).

The amount of chirp $\left(f_{h} / f_{l}\right)$ used in the wavelet has a direct correspondence to which area of the expansion is accented. A wavelet with $f_{h} / f_{l}=2$ will stress the area of the scalogram where the time interval of the LAPP signal has $f_{h} / f_{l} \approx 2$, near the end of the pulse. A ratio of $f_{h} / f_{l}=10$ will accent an area of the scalogram closer to $t=0$. In the non-chirp case $\left(f_{h} / f_{l}=1\right)$, the LAPP pulse is still apparent, 
but very weak in the spectrogram, since theoretically there are no time subintervals in the LAPP where there is constant frequency. Interestingly, at large values of $f_{h} / f_{l}$, we do not witness a marked decrease in performance, since the wavelets provide coverage of the LAPP signal at each level.

The wavelet transform employed here uses seven scale levels, resulting in a transform with $\approx 10 \%$ of the number of coefficients in the spectrogram of Figure (3). The number of scale levels required to capture the LAPP pulse can be further reduced by applying high values of $f_{h} / f_{l}$. In addition, the dyadic scaling linearizes the chirp. Thus, an appropriate template match to the LAPP signal is based on a straight line, as illustrated in the next section.

\section{Detection Results}

In order to detect the LAPP signals, we employed a template match based thresholding scheme. The detection algorithm proceeds as follows. In the pre-processing, a time/frequency template was constructed for the wavelet expansion, in the form of a straight line. Twenty five slopes were used, resulting in 25 total templates. For each slope value, eight different lags were selected, allowing the LAPP to be located at different points in the time interval. To normalize each line, its value was set equal to one, and the coefficients on either side of the line were weighted to form a triangular tunnel in three dimensional space. In the on-line processing, an inner product was formed between each resulting template and the absolute value of the scalogram. Next, the maximum of all the inner products was selected, and compared against a threshold in order to detect the LAPP signal.

The algorithm was tested on four different sets of LAPP signals, and also a data set containing noise and carriers but no LAPP signal. Forty five different values of $f_{h} / f_{l}$ were used, varying from 1 to approximately 400 . Only one false alarm was witnessed, $f_{h} / f_{l}=9.5$, which barely exceeded the threshold. The scheme performed the best at high values of $f_{h} / f_{i}$, with perfect detection at rates greater than 16. At lower values, the wavelet expansion will enhance isolated regions of the LAPP (as described in the previous section) resulting in "hit or miss" behavior of the detector. Thus, the worst detection levels are for values of $f_{h} / f_{l}<5$. Detection results are displayed in Table (1).

\section{Summary}

In this work, we have employed a discrete chirped wavelet transform and detection methods to find impulsive signals dispersed by the ionosphere in satellite data. Time/frequency methods were enlisted due to the difficulty detecting the signals in either domain. Actual data recorded by the US Department of Energy's ALEXIS/BLACKBEARD satellite were examined.

This work demonstrates that chirped wavelets, when combined with an appropriate template matching threshold detection scheme, can be utilized to detect nonlinear transionospheric signais under very low signal-to-noise ratios. Large rates of chirp showed superior detection properties, with perfect detection achieved for mother wavelets of $f_{h} / f_{l}>16$. More detailed results will be presented in the full paper.

\begin{tabular}{c|c|c|c|c|}
\cline { 3 - 5 }$f_{h} / f_{1}$ & $>16$ & $10-15$ & $5-10$ & $<5$ \\
\hline $\begin{array}{c}\text { percent } \\
\text { detection }\end{array}$ & 100 & 94 & 95 & 77 \\
\cline { 2 - 5 }
\end{tabular}

Table 1: Detection algorithm results for various values of $f_{h} / f_{l}$.

\section{References}

[1] R. Massey, "Impulse response function for a transionospheric signal through a Gaussian filter," Radio Science, vol. 28, pp643-647, 1993.

[2] S. Qian, M.E. Dunham, M.J. Freeman, "Transionospheric signal recognition by joint time-frequency representation," Radio Science, vol. 30, pp1817-1829, 1995.

[3] S. Mann, S. Haykin, "The Chirplet Transform: Physical Considerations," IEEE Transactions on Signal Processing, vol.43, no.11, pp.2745-2761, 1995.

[4] A. Doser, M. Dunham, "Efficient Transionospheric Signal Analysis Using Chirped Wavelets," submitted: 2nd IEEE UK Syposium on Applications of Time-Frequency and Time-Scale Analysis, Coventry, UK, Aug. 1997.

[5] S. Qian, D. Chen, Joint Time-Frequency Analysis: Methods and Applications, Prentice Hall, 1996. 


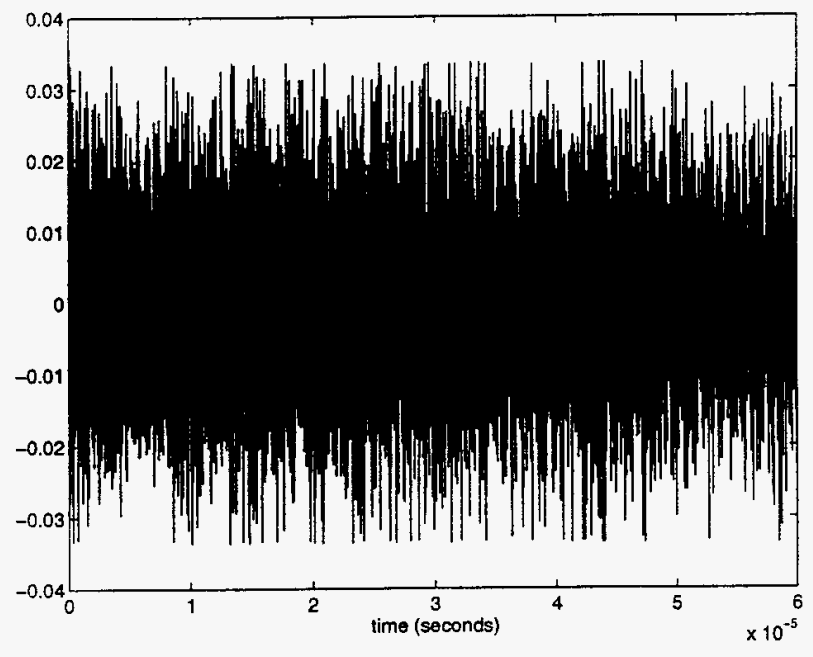

Figure 1: LAPP data signal, time series.

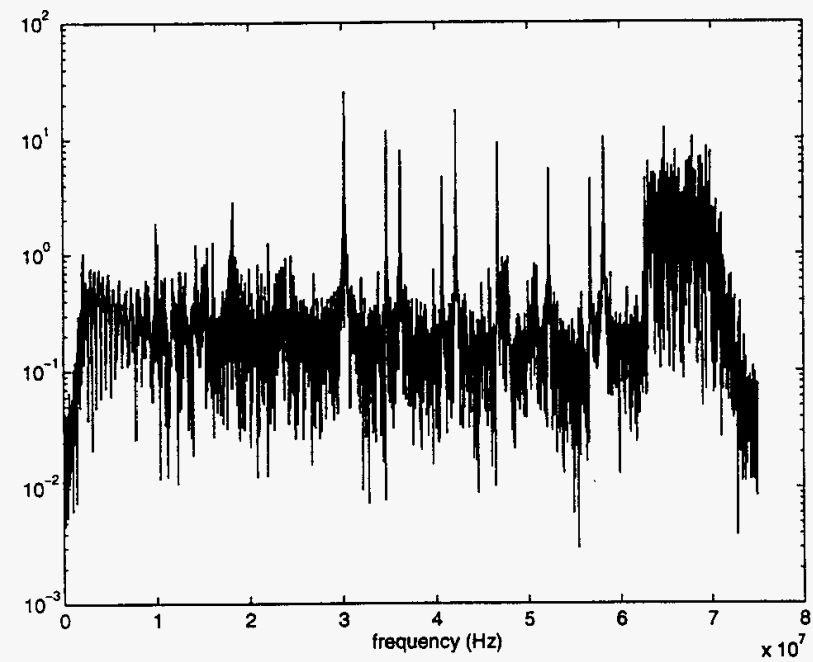

Figure 2: FFT of LAPP signal.

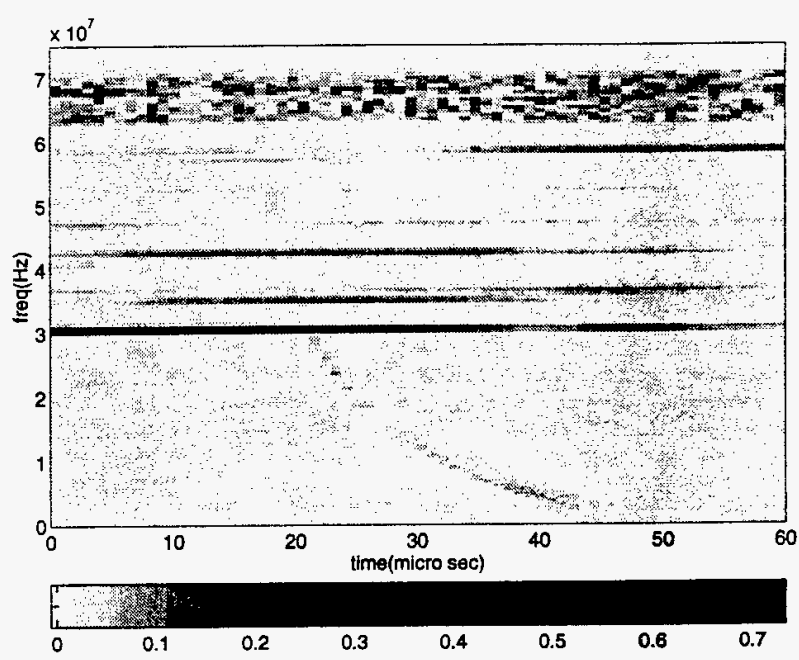

Figure 3: Spectrogram of LAPP data signal.

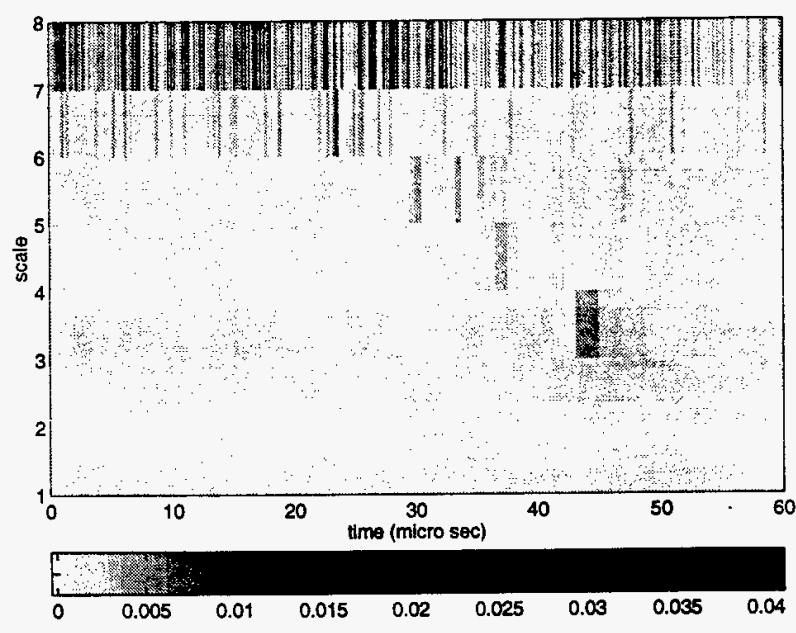

Figure 4: Wavelet Expansion of LAPP signal, $f_{h} / f_{l}=15$. 


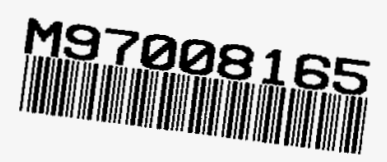

Report Number (14) $L A-U R--97-1855$

CONF-9711.54-

Publ. Date (11) 199707

Sponsor Code (18) DOD, XF

UC Category (19) UC-OOO,DOE/ER 